\section{Sollberger \\ P. Fuhr \\ Flexor myoclonus of the arm due to posttraumatic cervico-thoracic syringomyelia}

Received: 13 September 2007

Received in revised form: 28 December 2007 Accepted: 6 February 2008

Published online: 4 July 2008

Sirs: Syringomyelia rarely causes involuntary movements such as respiratory synkinesis, myoclonus, dystonia and tremor, probably due to an increased excitability of the spinal motor neurons [1]. We report the case of a pure pain-sensitive segmental myoclonus of the right arm in posttraumatic cervicothoracic syringomyelia, initially suspected to be a relapse of drugdependant behavior.

A 33-year-old man developed painful paresthesias and hypesthesia over the shoulder-girdle and in both arms 3 years after a complete transverse spinal cord injury at the fourth thoracic level. His past medical history was known for abuse of heroin until the age of 16 , chronic intake of methadone and an idiopathic hypogonadism substituted with testosterone. Within few months the sensory symptoms disappeared on the left side, whereas they increased on the right side. Moreover, repetitive involuntary jerky flexion movements of the right arm developed, involving predominantly the proximal arm muscles. They were exclusively triggered by spontaneous intense pain

M. Sollberger, MD (区) $\cdot$ P. Fuhr, MD

Dept. of Neurology

University Hospital of Basel

Petersgraben 4

4031 Basel, Switzerland

Tel.: +41612658930

Fax: +41 612653788

E-Mail: sollbergerm@uhbs.ch in the right arm as well as self-inflicted pressure-induced pain in the right upper arm. The severity of the jerky movements was strongly associated with the intensity of the pain. The movements could be absent for days or appear once a day for about a second up to several times within several hours. They were unaffected by voluntary contractions and not associated with amnesia or additional sensory-motor disturbances. Examination revealed a right-sided dominant hyporeflexia of the upper extremities and a paramedian right-sided dissociated sensory loss below the third cervical dermatome with sparing of the right hand. In addition there was the known complete transverse spinal cord syndrome at the 6 th thoracic level with sensory loss of all qualities, paresis of abdominal - and lower back musculature, paraplegia and bilateral extensor plantar reflexes. At the upper extremities there was no atrophy, no weakness and no impairment of coordination. Cranial nerves were intact. MRI of the cervical spine, performed 20 months before sensory symptoms started, was normal. MRI of the brain was normal. EEG showed an intermittent bifrontal slowing but no epileptiform potentials. Together with the intermittent nature of the jerks and the fluctuating sensory symptoms a psychogenic disorder or a relapse of drugdependent behavior was suspected. Increasing the dosage of methadone diminished pain and involuntary movements somewhat. In the following months the involuntary clonic flexor movement of the arm changed to a more dystonic flexor type without any associated changes in motor-sensory symptoms. Persistence of symptoms and increased disability of the patient led to a revaluation of the case. Median nerve sensory evoked potentials were normal, but cutaneous silent period (CSP) in the voluntary contracted right abductor pollicis brevis (APB) muscle was attenuated (Fig. 1). The patient refused any additional neurophysiological exams. Repeated MRI of the cervical spine now revealed an extensive syringomyelia from the second cervical segment down to the level of the spinal cord lesion at Th2/Th3 (Fig.2). After the syringo-peritoneal drainage, pain and involuntary movements decreased clearly within the first 2 weeks, followed by a stable clinical course ever since ( 9 months).

This case shows exclusively pain-sensitive segmental flexor myoclonus in an upper extremity, most likely caused by disinhibition of the flexor reflex due to posttraumatic cervico-thoracic syringomyelia. The marked decrease of the involuntary movements after surgical intervention together with the neurophysiological and imaging analyses point to the spinal origin of the myoclonus. Unfortunately no timelocked back-averaged EEG, no EMG polygraphs and no reflex studies other than CSP could be performed to better characterize the mechanism of the myoclonus. The case highlights the wide spectrum of involuntary movements reported in patients with syringomyelia, i.e. those which are spontaneous and stimulus-dependant $[1,2,4]$, spontaneous and stimulus-independent [3] or, as in our case, exclusively stimulus-dependent [2]. Interestingly, in spite the clinical heterogeneity a similar pathogenic mechanism is postulated, which is increased excitability of spinal motor neurons of probably different intensity due to altered processing at the inhibitory spinal interneuron circuits [1]. Of note in our case is the change of the clonic flexor movement to a more dystonic flexor type. That change in movement implicates longer lasting excitatory periods of 
Fig. 1 CSP in the right APB of a healthy control (a) and the patient (b). Stimulation of second digit at $13 \times$ sensory threshold (ST) in (a) and at $16 \times \mathrm{ST}$ in (b) (arrow). Stimulus duration $0.2 \mathrm{~ms}$. Average EMG bases on the period of EMG activity prior to the stimulus

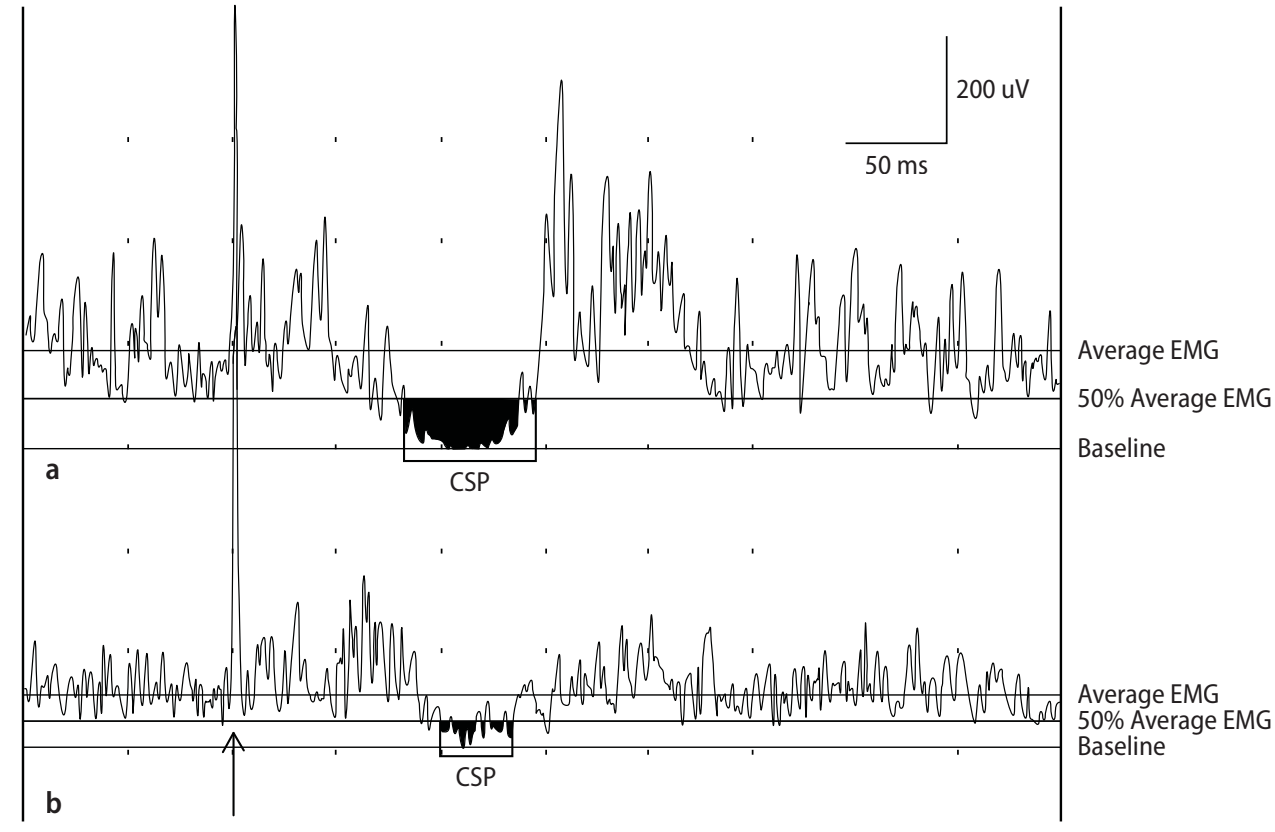

In the present case, posterior columns as assessed by median nerve sensory evoked potentials were normal, while CSP, an inhibitory part of the flexor reflex, in the right hand was attenuated. Afferent impulses that generate the CSP are carried by A-delta fibers, which conduct nociceptive impulses on spinal interneurons, located in close proximity to the central canal $[5,6,12]$. These findings, together with the phenomenology of the myoclonus and its response to methadone, suggest a mechanism involving an intraspinal pathway, susceptible to nociceptive input and endorphin receptor activation. There is evidence from human and animal literature that endogenous and exogenous opioids suppress spinal nociceptive flexion reflexes mainly by acting on spinal mu-opioid receptors [7-9]. However, except in patients with restless legs syndrome, respectively periodic limb movements during sleep [10] opioids are not known to decrease involuntary movements of spinal origin. In contrary, myoclonus has been described as a neuroexcitatory side effect of opioids [11]. In this regard we report an unusual pharmacological effect of methadone on spinal myoclonus, which, however, goes with the hypothesis of a disinhibited spinal flexor reflex.

It is of note that CSP is also altered in patients with syringomyelia without involuntary movements [12,13], which implies an additional mechanism for the painassociated motor release phenomenon in the reported case.

\section{References}

1. Nogués MA, Leiguarda RC, Rivero AD, et al. (1999) Involuntary movements and abnormal spontaneous EMG activity in syringomyelia and syringobulbia. Neurology 52:823-834

2. Hill MD, Kumar R, Lozano A, et al. (1999) Syringomyelic dystonia and athetosis. Movement Disorders 14: 684-688

3. Bagnato S, Rizzo V, Quartarone A, et al. (2001) Segmental myoclonus in a patient affected by syringomyelia. Neurol Sci 22:27-29

4. Gregoire SM, Laloux P, Hanson P, et al. (2006) Segmental spinal myoclonus and syringomyelia: A case report. Acta Neurol Belg 106:37-40

5. Floeter MK (2003) Cutaneous silent periods. Muscle Nerve 28:391-401 spinal motor neurons [1] probably the syrinx. 
6. Kofler M, Fuhr P, Leis AA, et al. (2001) Modulation of upper extremity motor evoked potentials by cutaneous afferents in humans. Clin Neurophysiol 112: 1053-1063

7. Willer JC, Dehen H, Cambier J (1981) Stress-induced analgesia in humans: endogenous opioids and naloxone-reversible depression of pain reflexes. Science 212:689-691

8. Willer JC (1985) Studies on pain. Effects of morphine on a spinal nociceptive flexion reflex and related pain sensation in man. Brain Res 331:105-114
9. Tao PL, Lai YS, Chow LH, et al. (2005) Effects of morphine and endomorphins on the polysynaptic reflex in the isolated rat spinal cord. Naunyn Schmiedebergs Arch Pharmacol 371: $72-80$

10. Karatas M (2007) Restless legs syndrome and periodic limb movements during sleep: diagnosis and treatment. Neurologist 13:294-301
11. Glare P, Walsh D, Sheehan D (2006) The adverse effects of morphine: a prospective survey of common symptoms during repeated dosing for chronic cancer pain. Am J Hosp Palliat Care 23:229-235

12. Stetkarova I, Kofler M, Leis AA (2001) Cutaneous and mixed nerve silent periods in syringomyelia. Clin Neurophysiol 112:78-85

13. Kaneko K, Kawai S, et al. (1997) Cutaneous silent period in syringomyelia. Muscle Nerve 20:884-886 